\title{
Erratum to: Differences between amyloid toxicity in alpha and beta cells in human and mouse islets and the role of caspase- 3
}

\author{
E. Law • S. Lu • T. J. Kieffer • G. L. Warnock • Z. Ao • \\ M. Woo $\cdot$ L. Marzban
}

Published online: 6 July 2013

(C) Springer-Verlag Berlin Heidelberg 2013

Erratum to: Diabetologia

DOI 10.1007/s00125-010-1717-9

Unfortunately in the second row of Fig. $4 \mathrm{c}$ the islet shown in the micrograph for $\mathrm{h} I A P P^{-} / \mathrm{Casp}^{+}$(third column) was accidentally duplicated in the second column for $\mathrm{h} I A P P^{+} / \mathrm{Casp}^{-}$. Figure $4 \mathrm{c}$ is reproduced overleaf, with the correct micrograph for $\mathrm{h} I A P P^{+} /$Casp $^{-}$in column 2 . The authors regret this oversight and confirm that this error has no impact on the data presented, interpreted or discussed in the article.

The online version of the original article can be found at http://dx.doi.org/ 10.1007/s00125-010-1717-9.

E. Law $\cdot \mathrm{S} . \mathrm{Lu} \cdot \mathrm{L}$. Marzban

Division of Pharmacology and Toxicology,

Faculty of Pharmaceutical Sciences,

University of British Columbia,

Vancouver, Canada

\section{T. J. Kieffer}

Department of Cellular and Physiological Sciences,

Faculty of Medicine, University of British Columbia,

Vancouver, BC, Canada

T. J. Kieffer · G. L. Warnock $\cdot$ Z. Ao

Department of Surgery, Faculty of Medicine,

University of British Columbia,

Vancouver, BC, Canada

M. Woo

Department of Medicine, University of Toronto,

Ontario Cancer Institute, St Michael's Hospital,

Toronto, ON, Canada

Present address:

L. Marzban $(\bowtie)$

Department of Surgery, Faculty of Medicine,

University of British Columbia, Jim Pattison Pavilion,

Vancouver General Hospital,

910 W 10th Avenue,

Vancouver, BC, Canada V5Z 4E3

e-mail: lucy.marzban@ubc.ca 
C
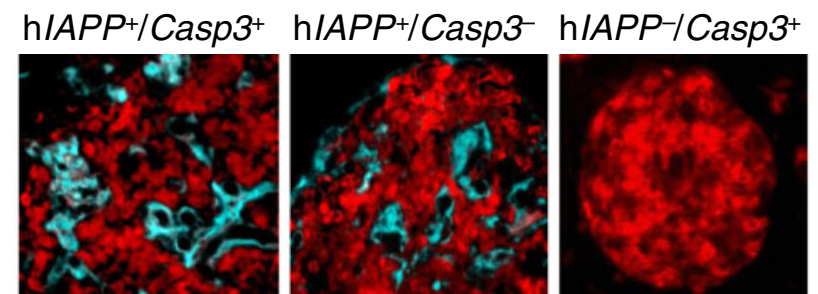

$\mathrm{h} / A P P^{-} /$Casp3
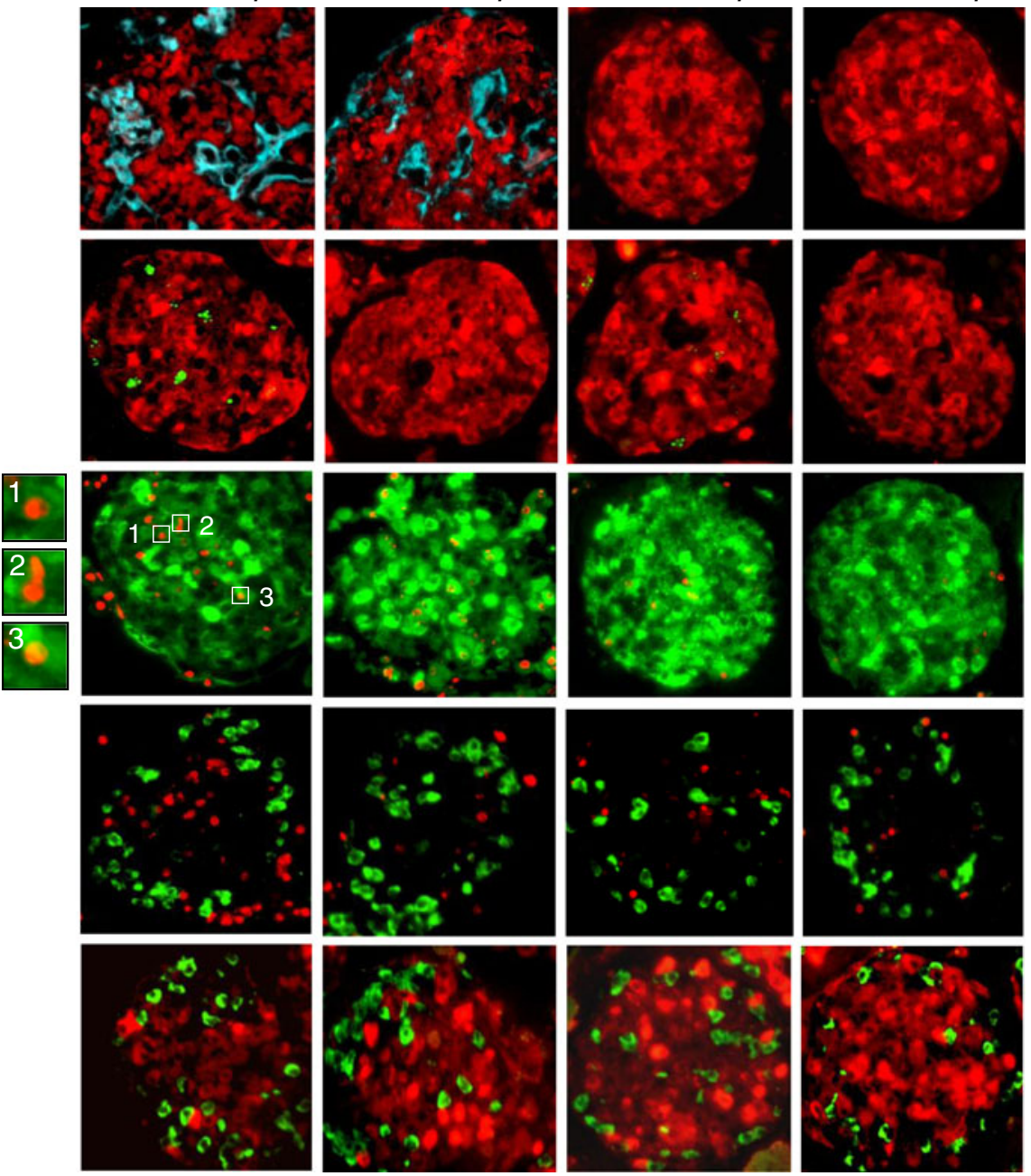

Fig. 4 c Islet sections of 7 day cultured islets from hIAPP-transgenic (red) and active caspase-3 (green); insulin (green) and TUNEL (red); mice (8-16 weeks) expressing or lacking caspase- 3 were double stained (top to bottom) for insulin (red) and thioflavin S (bright blue); insulin glucagon (green) and TUNEL (red); or insulin (red) and glucagon (green) 